\title{
Electromagnetic Field in Higher-Dimensional Black-Hole Spacetimes*
}

\author{
Pavel Krtous \\ Institute of Theoretical Physics, \\ Faculty of Mathematics and Physics, \\ Charles University in Prague, \\ V Holešovičkách 2, Prague, Czech Republic
}

(Dated: June 28, 2007)

\begin{abstract}
A special test electromagnetic field in the spacetime of the higher-dimensional generally rotating NUT-(A)dS black hole is found. It is adjusted to the hidden symmetries of the background represented by the principal Killing-Yano tensor. Such electromagnetic field generalizes the field of charged black hole in four dimensions. In higher dimensions, however, the gravitational back reaction of such a field cannot be consistently solved.
\end{abstract}

PACS numbers: 04.50.+h, 04.70.Bw, 04.40.Nr, 04.20.Jb

\section{INTRODUCTION}

In recent years people have become interested in higher-dimensional spacetimes with motivation, among others, coming from string theories. One of the important classes of exact solutions of the Einstein equations are the spacetimes representing black holes. In the last years various generalizations of black hole solutions to the higher-dimensional gravity have been discovered. Starting with nonrotating and rotating black holes [1, 2] and following with the metric describing the rotating black hole with the cosmological constant in five dimensions [3], the generally rotating black hole in arbitrary dimension with the cosmological constant was found in [4, 5]. In 2006 this solution was rewritten [ [6] in much more convenient coordinates which allowed to add NUT parameters and compute the curvature tensors explicitly [7].

Recently, the properties of this solution have been discussed in various papers. It has been shown that the spacetime possesses hidden symmetries which can be described by the principal Killing-Yano tensor [8], the properties of which have been thoroughly discussed in [9]. One of the main consequences of these hidden symmetries is a possibility to find a complete set of integration constants for a geodesic motion which are in involution [9, 10, 11]. This result is related to the the separability of the Hamilton-Jacobi equation which, together with the separability of the Klein-Gordon equation, was demonstrated in [12].

It seems that a generalization of the black hole solution to include an electromagnetic field is not straightforward. Some partial results are know [13, 14, 15, 16, 17, 18], however a solution representing a rotating black hole with

\footnotetext{
*The results contained in this paper have been presented at the Black hole VI conference in White Point, Canada, May 12-16 2007, and at the GRG18 conference in Sydney, Australia, July 8-13 2007. ${ }^{\dagger}$ Electronic address: Pavel.Krtous@mff.cuni.cz
}

electromagnetic field parameterized by full number of black hole parameters and electric/magnetic charges is not known.

In this work we present the test electromagnetic field on the background described by the metric with the structure of black hole solution [6], however, we do not enforce the specific form of the metric function $X_{\mu}$ which is needed to satisfy the vacuum Einstein equations. The electromagnetic field is described by $n=\lfloor D / 2\rfloor$ electric and magnetic charges and it is adjusted to the explicit as well as hidden symmetries of the spacetime. In even dimensions our field is equivalent to the harmonic forms independently found in [19], our results supply the proof that these forms are harmonic in all even dimensions.

We also show that our field is a generalization of the electromagnetic field known from the Carter [20] and Plebański-Demiański 21] form of the black hole solution in $D=4$. In this special case, however, the metric function can be modified in such a way that that the metric and the electromagnetic field satisfy the full coupled Einstein-Maxwell equations. Unfortunately, the same is not true in the higher-dimensional case.

The plan of the paper is following. We discuss separately even and odd spacetime dimensions - although our procedure is very similar in both cases, the resulting expressions are slightly different and it would be artificial to try to 'squeeze' them into the same equations. We discuss the case of even dimensions in detail and than sketch the corresponding results for the odd dimensions. First we review the metric and the symmetries of the black hole spacetime, next we find the test electromagnetic field 'adjusted' to the symmetries of the spacetime. For even dimensions we also discuss the case of the physical dimension $D=4$, in the odd dimension case we study the Chern-Simons modification of the electromagnetic field. Finally, we discuss a possibility to generalize our fields to a solution of the coupled Einstein-Maxwell equations. The results are recapitulated in the Summary and the paper is concluded by the technical Appendix. 


\section{EVEN DIMENSIONS, $D=2 n$}

\section{The metric}

We start with a simpler case of even spacetime dimension $D=2 n$. The spacetime of generally rotating black hole with NUT charges can be described by the metric

$$
\boldsymbol{g}=\sum_{\mu=1}^{n}\left[\frac{U_{\mu}}{X_{\mu}} \boldsymbol{d} x_{\mu}{ }^{2}+\frac{X_{\mu}}{U_{\mu}}\left(\sum_{k=0}^{n-1} A_{\mu}^{(k)} \boldsymbol{d} \psi_{k}\right)^{2}\right] .
$$

Here $x_{\mu}, \mu=1, \ldots, n$, correspond to radial $^{1}$ and latitudinal directions, while $\psi_{j}, j=0, \ldots, n-1$, to temporal and longitudinal directions. The functions $U_{\mu}$ and $A_{\mu}^{(k)}$ (together with $U$ and $A^{(k)}$ used below) are defined as

$$
\begin{gathered}
U_{\mu}=\prod_{\substack{\nu=1 \\
\nu \neq \mu}}^{n}\left(x_{\nu}^{2}-x_{\mu}^{2}\right), \quad U=\prod_{\substack{\mu, \nu=1 \\
\mu<\nu}}^{n}\left(x_{\mu}^{2}-x_{\nu}^{2}\right), \\
A_{\mu}^{(k)}=\sum_{\substack{\nu_{1}, \ldots, \nu_{k}=1 \\
\nu_{1}<\cdots<\nu_{k}, \nu_{i} \neq \mu}}^{n} x_{\nu_{1}}^{2} \ldots x_{\nu_{k}}^{2}, \quad A^{(k)}=\sum_{\substack{\nu_{1}, \ldots, \nu_{k}=1 \\
\nu_{1}<\cdots<\nu_{k}}}^{n} x_{\nu_{1}}^{2} \ldots x_{\nu_{k}}^{2} .
\end{gathered}
$$

We call the remaining functions $X_{\mu}$ the metric functions.

In the following we consider a broader class of spacetimes than merely the black hole spacetime, namely we just assume that the metric functions $X_{\mu}$ depend arbitrarily on a single coordinate, $X_{\mu}=X_{\mu}\left(x_{\mu}\right)$. For the black hole spacetime they acquire a specific from (see (2.10) below) determined by the vacuum Einstein equations.

The metric can be diagonalized

$$
\boldsymbol{g}=\sum_{\mu=1}^{n}\left(\frac{U_{\mu}}{X_{\mu}} \boldsymbol{\epsilon}^{\mu} \boldsymbol{\epsilon}^{\mu}+\frac{X_{\mu}}{U_{\mu}} \boldsymbol{\epsilon}^{\hat{\mu}} \boldsymbol{\epsilon}^{\hat{\mu}}\right)=\sum_{a=1}^{D} \boldsymbol{e}^{a} \boldsymbol{e}^{a}
$$

introducing the unnormalized frame $\epsilon^{a}$ and normalized frame $\boldsymbol{e}^{a}$ of 1 -forms

$$
\begin{array}{rlrl}
\boldsymbol{\epsilon}^{\mu} & =\boldsymbol{d} x_{\mu}, & \boldsymbol{e}^{\mu} & =\left[\frac{U_{\mu}}{X_{\mu}}\right]^{1 / 2} \boldsymbol{\epsilon}^{\mu}, \\
\boldsymbol{\epsilon}^{\hat{\mu}} & =\sum_{k=0}^{n-1} A_{\mu}^{(k)} \boldsymbol{d} \psi_{k}, & \boldsymbol{e}^{\hat{\mu}}=\left[\frac{X_{\mu}}{U_{\mu}}\right]^{1 / 2} \boldsymbol{\epsilon}^{\hat{\mu}} .
\end{array}
$$

The dual frames of vectors are given by

$$
\begin{array}{ll}
\boldsymbol{\epsilon}_{\mu}=\boldsymbol{\partial}_{x_{\mu}}, & \boldsymbol{e}_{\mu}=\left[\frac{X_{\mu}}{U_{\mu}}\right]^{1 / 2} \boldsymbol{\epsilon}_{\mu}, \\
\boldsymbol{\epsilon}_{\hat{\mu}}=\sum_{k=0}^{n-1} \frac{\left(-x_{\mu}^{2}\right)^{n-1-k}}{U_{\mu}} \boldsymbol{\partial}_{\psi_{k}}, & \boldsymbol{e}_{\hat{\mu}}=\left[\frac{U_{\mu}}{X_{\mu}}\right]^{1 / 2} \boldsymbol{\epsilon}_{\hat{\mu}} .
\end{array}
$$

\footnotetext{
1 The radial coordinate (and some other related quantities) are actually rescaled by the imaginary unit $i$ in order to put the metric to a more symmetric and compact form - cf., e.g., [6].
}

Here we use the convention $\hat{\mu}=\mu+n$ and Greek indieces run from 1 to $n$. The inverse relations can be easily obtained with help of the relations (A1) in the Appendix.

Let us note that the definition of unnormalized 1-forms $\boldsymbol{\epsilon}^{a}$ and vectors $\boldsymbol{\epsilon}_{a}$ does not depend on the metric functions $X_{\mu}$.

It was shown in [7] that the Ricci curvature for such a metric is

$$
\mathbf{R i c}=-\sum_{\mu=1}^{n} r_{\mu}\left(e^{\mu} e^{\mu}+e^{\hat{\mu}} e^{\hat{\mu}}\right)
$$

where

$$
\begin{aligned}
r_{\mu} & =\frac{1}{2} \frac{X_{\mu}^{\prime \prime}}{U_{\mu}}+\sum_{\substack{\nu=1 \\
\nu \neq \mu}}^{n} \frac{1}{U_{\nu}} \frac{x_{\nu} X_{\nu}^{\prime}-x_{\mu} X_{\mu}^{\prime}}{x_{\nu}^{2}-x_{\mu}^{2}}-\sum_{\substack{\nu=1 \\
\nu \neq \mu}}^{n} \frac{1}{U_{\nu}} \frac{X_{\nu}-X_{\mu}}{x_{\nu}^{2}-x_{\mu}^{2}} \\
& =\frac{\partial}{\partial x_{\mu}^{2}}\left[\sum_{\nu=1}^{n} \frac{x_{\nu}^{2}\left(x_{\nu}^{-1} X_{\nu}\right)_{, \nu}}{U_{\nu}}\right] .
\end{aligned}
$$

The scalar curvature simplifies to

$$
\mathcal{R}=-\sum_{\nu=1}^{n} \frac{X_{\nu}^{\prime \prime}}{U_{\nu}}
$$

Here, the primes denote the differentiation with respect to the single argument of the metric function, $X_{\mu}^{\prime}=X_{\mu, \mu}$.

The requirement that the metric (2.1) satisfies the Einstein equations with the cosmological constant implies that the metric functions have the form

$$
X_{\mu}=b_{\mu} x_{\mu}+\sum_{k=0}^{n} c_{k} x_{\mu}^{2 k},
$$

see [6, 7] or (A6) in the Appendix. The constants $c_{k}$ and $b_{\mu}$ are then related to the cosmological constants, angular momenta, mass, and NUT charges, see, e.g., [6] for details. For $b_{\mu}=0$ we obtain 7$]$ the constant curvature spacetime with the scalar curvature $\mathcal{R}=-2 n(2 n-1) c_{2 n}$.

The spacetime with the metric (2.1) possesses the explicit symmetries given by the Killing vectors $\boldsymbol{\partial}_{\psi_{j}}$ and hidden symmetries which are related to the principal Killing-Yano tensor discovered in [8] and discussed in detail in [9]. The principal Killing-Yano tensor is dual to the rank-2 closed conformal Killing-Yano tensor which has a very simple form in the frames $\boldsymbol{\epsilon}^{a}$ and $\boldsymbol{e}^{a}$ :

$$
\boldsymbol{h}=\sum_{\mu=1}^{n} x_{\mu} \boldsymbol{\epsilon}^{\mu} \wedge \boldsymbol{\epsilon}^{\hat{\mu}}=\sum_{\mu=1}^{n} x_{\mu} \boldsymbol{e}^{\mu} \wedge \boldsymbol{e}^{\hat{\mu}} .
$$

It has been demonstrated in [9] that it is possible to generate a series of higher-rank Killing-Yano tensors and a series of rank-2 Killing tensors from $\boldsymbol{h}$. The conformal Killing-Yano tensor also identifies eigenspaces spanned on the pairs $\left\{\boldsymbol{e}_{\mu}, \boldsymbol{e}_{\hat{\mu}}\right\}$ and coordinates $x_{\mu}$ as corresponding eigenvalues. 


\section{Algebraically special test electromagnetic field}

Now we turn to the task to find an algebraically special test electromagnetic fields on the background given by the metric (2.1). By algebraically special we mean that the Maxwell tensor $\boldsymbol{F}$ shares the explicit symmetry of the metric (it is independent of $\psi_{j}$ ) and it is aligned with the hidden symmetry of the spacetime, namely it has the same eigenspaces as the principal conformal Killing-Yano tensor $\boldsymbol{h}$. We thus require

$$
\boldsymbol{F}=\sum_{\mu=1}^{n} f_{\mu} \boldsymbol{\epsilon}^{\mu} \wedge \boldsymbol{\epsilon}^{\hat{\mu}}, \quad f_{\mu}=f_{\mu}\left(x_{1}, \ldots, x_{n}\right) .
$$

The Maxwell tensor is generated by the vector potential, $\boldsymbol{F}=\boldsymbol{d} \boldsymbol{A}$. We assume the vector potential

$$
\boldsymbol{A}=\sum_{\mu=1}^{n}\left(A_{\mu} \boldsymbol{\epsilon}^{\mu}+A_{\hat{\mu}} \boldsymbol{\epsilon}^{\hat{\mu}}\right)
$$

with the components $A_{\mu}$ and $A_{\hat{\mu}}$ independent of $\psi_{j}$. Comparing $\boldsymbol{d} \boldsymbol{A}$ with (2.12) we find that $A_{\mu}$ terms are gauge-trivial and $A_{\hat{\mu}}$ must satisfy $\left(\left(x_{\nu}^{2}-x_{\mu}^{2}\right) A_{\hat{\mu}}\right)_{, \nu}=0$, from which follows that the vector potential can be written as

$$
\boldsymbol{A}=\sum_{\mu=1}^{n} \frac{g_{\mu} x_{\mu}}{U_{\mu}} \boldsymbol{\epsilon}^{\hat{\mu}}
$$

where $g_{\mu}$ are functions of a single variable only, $g_{\mu}=g_{\mu}\left(x_{\mu}\right)$. Evaluating the Maxwell tensor we get the components $f_{\mu}$ :

$$
f_{\mu}=\frac{g_{\mu}}{U_{\mu}}+\frac{x_{\mu} g_{\mu}^{\prime}}{U_{\mu}}+2 x_{\mu} \sum_{\substack{\nu=1 \\ \nu \neq \mu}}^{n} \frac{1}{U_{\nu}} \frac{x_{\nu} g_{\nu}-x_{\mu} g_{\mu}}{x_{\nu}^{2}-x_{\mu}^{2}}
$$

Alternatively, we could apply directly the first Maxwell equation $\boldsymbol{d} \boldsymbol{F}=0$ to the Maxwell tensor (2.12). With help of identity w5e find that $f_{\mu}$ are generated by an auxiliary potential $\phi$,

$$
f_{\mu}=\phi_{, \mu}
$$

which satisfies the equation

$$
\phi_{, \mu \nu}=2 \frac{x_{\nu} \phi_{, \mu}-x_{\mu} \phi_{, \nu}}{x_{\mu}^{2}-x_{\nu}^{2}} \quad \text { for } \mu \neq \nu .
$$

The field (2.14) found above is generated by the potential

$$
\phi=\sum_{\nu=1}^{n} \frac{g_{\nu} x_{\nu}}{U_{\nu}}
$$

Next we proceed to calculate the source $\boldsymbol{J}$ of the electromagnetic field using the second Maxwell equation $\boldsymbol{J}=-\boldsymbol{\nabla} \cdot \boldsymbol{F}$. Expressing the Maxwell tensor in coordinates $x_{\mu}, \psi_{j}$, using $F_{; n}^{n a}=\mathfrak{g}^{-1 / 2} \sum_{\nu}\left(\mathfrak{g}^{1 / 2} F^{\nu a}\right)_{, \nu}$, the fact that the determinant $\mathfrak{g}$ of the metric in these coordinates is $\mathfrak{g}=U^{2}$, identities (A1), (A2), and relations (2.12), (2.16) we obtain

$$
\boldsymbol{J}=\sum_{\mu=1}^{n} j_{\mu} \boldsymbol{\epsilon}_{\hat{\mu}}
$$

with

$$
j_{\mu}=-2 \frac{\partial}{\partial x_{\mu}^{2}}\left[\phi-x_{\mu}^{2} \sum_{\nu=1}^{n} x_{\nu}^{-1} \phi_{, \nu}\right] .
$$

Substituting (2.18) we finally obtain

$$
j_{\mu}=2 \frac{\partial}{\partial x_{\mu}^{2}}\left[\sum_{\nu=1}^{n} \frac{x_{\nu}^{2} g_{\nu}^{\prime}}{U_{\nu}}\right] .
$$

It is worth to mention that the expression for the source $\boldsymbol{J}$ does not contain any reference to the metric functions $X_{\mu}$.

We are interested in the electromagnetic field without sources, so we require $\boldsymbol{J}=0$. Integrating (2.21) we find that the sum in the square brackets has to be a constant. However, this sum has a special form discussed in the Appendix. Using (A6) we find that $g_{\mu}^{\prime}$ are given by a single polynomial of the $(n-1)$-th order in variable $x_{\mu}^{2}$. Integrating once more we find

$$
g_{\mu} x_{\mu}=e_{\mu} x_{\mu}+\sum_{k=0}^{n-1} a_{k}\left(-x_{\mu}^{2}\right)^{n-1-k} .
$$

Substituting into the vector potential (2.14) or the scalar potential (2.18) we find with help of the relations (A1) that the terms containing the constants $a_{k}$ are gauge trivial (they contribute by $\sum_{k=0}^{n-1} a_{k} \boldsymbol{d} \psi_{k}$ into $\boldsymbol{A}$ or by just the constant $a_{0}$ into $\phi$ ) and they can be ignored.

We thus have found that the algebraically special electromagnetic field (i.e., the field of the form (2.12) ) satisfies the Maxwell equations on the background described by the metric (2.1) if and only if it is generated by the vector potential

$$
\boldsymbol{A}=\sum_{\mu=1}^{n} \frac{e_{\mu} x_{\mu}}{U_{\mu}} \boldsymbol{\epsilon}^{\hat{\mu}}
$$

The components $f_{\mu}$ of the Maxwell tensor are easily determined by (2.16) from the auxiliary potential

$$
\phi=\sum_{\mu=1}^{n} \frac{e_{\mu} x_{\mu}}{U_{\mu}}
$$

and they are

$$
f_{\mu}=\frac{e_{\mu}}{U_{\mu}}+2 x_{\mu} \sum_{\substack{\nu=1 \\ \nu \neq \mu}}^{n} \frac{1}{U_{\nu}} \frac{x_{\nu} e_{\nu}-x_{\mu} e_{\mu}}{x_{\nu}^{2}-x_{\mu}^{2}}
$$


Here, $e_{\mu}$ are constants which can be related using the Gauss and Stokes theorems to the electric and magnetic charges of the field.

If we set all charges except one, say $e_{\nu}$, to zero, the Maxwell tensor $\boldsymbol{F}$ corresponds to the harmonic form $\boldsymbol{G}_{(2)}^{(\nu)}$ recently found and verified for particular cases in [19].

The surprising property of our field is that it satisfies the Maxwell equations independently of a specific form of the metric functions $X_{\mu}$. Moreover, as we will see in (2.30), the stress-energy tensor corresponding to the field (2.12) has the form consistent with the structure of the Ricci (and the Einstein) tensor (2.7). These facts open a possibility that we could solve the full Einstein-Maxwell equations: modifying the metric functions $X_{\mu}$ we could construct the spacetime in which the stress-energy tensor $\boldsymbol{T}$ would be a source for the Einstein equations, and the electromagnetic field would remain the solution of the Maxwell equations.

First, we will show that this goal can be achieved in $D=4$ dimensions. Unfortunately, next we will demonstrate that this procedure does not work in higher dimensions.

\section{Case $D=4$}

The metric (2.1) is a generalization of the $D=4$ black hole solution in the form found by Carter 20] and elaborated by Plebański and Demiański [21]. The full class of Plebański-Demiański solutions includes accelerated rotating NUT and electromagnetically charged black holes. The meaning of all parameters of the solutions was recently discussed in the series of papers $22,23,24,25,26$. We use the charged Plebański-Demiański metric with the acceleration set to zero: ${ }^{2}$

$$
\begin{aligned}
\boldsymbol{g}= & -\frac{Q}{r^{2}+p^{2}}\left(\boldsymbol{d} t+p^{2} \boldsymbol{d} \sigma\right)^{2}+\frac{r^{2}+p^{2}}{Q} \boldsymbol{d} r^{2} \\
& +\frac{r^{2}+p^{2}}{P} \boldsymbol{d} p^{2}+\frac{P}{r^{2}+p^{2}}\left(\boldsymbol{d} t-r^{2} \boldsymbol{d} \sigma\right)^{2},
\end{aligned}
$$

where

$$
\begin{aligned}
& Q=e^{2}-2 m r+k+\varepsilon r^{2}+\lambda r^{4}=\mathcal{X}\left(-r^{2}\right)-2 m r+e^{2}, \\
& P=-g^{2}+2 n p+k-\varepsilon p^{2}+\lambda p^{4}=\mathcal{X}\left(p^{2}\right)+2 n p-g^{2} .
\end{aligned}
$$

The electromagnetic field is given by

$$
\boldsymbol{A}=-\frac{1}{r^{2}+p^{2}}\left(e r\left(\boldsymbol{d} t+p^{2} \boldsymbol{d} \sigma\right)+g p\left(\boldsymbol{d} t-r^{2} \boldsymbol{d} \sigma\right)\right) .
$$

Here $t, r, p, \sigma$ are temporal, radial, latitudinal, and longitudinal coordinates, $m, n, \varepsilon, \lambda$ are parameters related to

\footnotetext{
${ }^{2}$ See, e.g., eqs. (5) and (6) of [24] with acceleration $\alpha=0$. Here we also used the gauge freedom to set $\omega=1$ and redefined $k$.
}

mass, NUT charge, angular momentum, and cosmological constant, and $e, g$ are electric and magnetic charges.

This metric and the electromagnetic field satisfy coupled Einstein-Maxwell equations. For $e, g=0$ the metric (2.26) satisfies the vacuum Einstein equations, however, even in this case the electromagnetic field (2.28) (with non-zero charges) satisfies the Maxwell equations and it is thus a valid test electromagnetic field on the given vacuum background.

Now, we can easily identify the Plebański-Demiański $\boldsymbol{g}$ and $\boldsymbol{A}$ with our general metric (2.1) and field (2.23) using the dictionary

$$
\begin{aligned}
& \psi_{0}=t, x_{1}=i r, X_{1}=Q, U_{1}=r^{2}+p^{2}, \quad e_{1}=i e, \\
& \psi_{1}=\sigma, x_{2}=p, X_{2}=P, U_{2}=-r^{2}-p^{2}, e_{2}=g .
\end{aligned}
$$

Clearly, if the charges $e, g$ are missing in the metric functions $Q$ and $P$, these functions corresponds exactly to $X_{1}$ and $X_{2}$ given by (2.10) and the electromagnetic field (2.28) corresponds to the test field given by (2.23). However, it is possible to modify $P$ and $Q$ by adding $e^{2}$ and $-g^{2}$ respectively (i.e., changing the vacuum $X_{\mu}$ 's by adding $-e_{\mu}^{2}$ ), and we obtain the metric and the electromagnetic field satisfying the coupled Einstein-Maxwell equations.

\section{Einstein-Maxwell equations in even $D \neq 4$}

In a generic dimension we first evaluate the stressenergy tensor $\boldsymbol{T}$ of the electromagnetic field (2.12). A straightforward calculation leads to

$$
8 \pi \boldsymbol{T}=\sum_{\mu=1}^{n}\left(2 f_{\mu}^{2}-f^{2}\right)\left(\boldsymbol{e}^{\mu} \boldsymbol{e}^{\mu}+\boldsymbol{e}^{\hat{\mu}} \boldsymbol{e}^{\hat{\mu}}\right),
$$

with the trace

$$
8 \pi T=2(2-n) f^{2},
$$

where the function $f^{2}$ is defined as

$$
f^{2}=\sum_{\nu=1}^{n} f_{\nu}^{2} .
$$

We explicitly see that the trace of the stress-energy is non-vanishing for $D \neq 4$ which is related to the fact that the electromagnetic field is not conformally invariant in a general dimension.

Now we would like to solve the Einstein equations Ric $-\frac{1}{2} \mathcal{R} \boldsymbol{g}+\Lambda \boldsymbol{g}=8 \pi \boldsymbol{T}$. The trace gives the condition

$$
\mathcal{R}=2 \frac{D}{D-2} \Lambda+2 \frac{D-4}{D-2} f^{2} .
$$

However, the scalar curvature has the form (2.9) and it immediately follows that

$$
\frac{\partial^{2 n-2}}{\partial x_{\mu}^{2 n-2}}\left(U_{\mu} \mathcal{R}\right)=-X_{\mu}^{[2 n]}
$$


which is a function of $x_{\mu}$ only. Applying this to the righthand-side of (2.33) we obtain the condition

$$
\frac{\partial^{2 n-2}}{\partial x_{\mu}^{2 n-2}}\left(U_{\mu} f^{2}\right) \quad \text { must be a function of } x_{\mu} \text { only. }
$$

It was checked by Mathematica that this conditions does not hold for the electromagnetic field given by (2.25), at least for the lowest non-trivial values of $n$. It seems that the main problem is that $\mathcal{R}$ behaves as $\sum h_{\mu} / U_{\mu}$ while $f^{2}$ as a square of such sums.

We thus may conclude that in a generic even dimension the electromagnetic field of the form (2.12), (2.25) cannot couple to the metric given by (2.1).

\section{ODD DIMENSIONS, $D=2 n+1$}

\section{The metric}

Let us briefly review modifications which appear in the odd dimensional case. We have an additional coordinate $\psi_{n}$ which labels an 'unpaired' angular direction. The metric contains an additional term,

$$
\begin{aligned}
\boldsymbol{g}=\sum_{\mu=1}^{n}\left[\frac{U_{\mu}}{X_{\mu}} \boldsymbol{d}_{\mu}^{2}+\frac{X_{\mu}}{U_{\mu}}\right. & \left.\left(\sum_{k=0}^{n-1} A_{\mu}^{(k)} \boldsymbol{d} \psi_{k}\right)^{2}\right] \\
& +\frac{c}{A^{(n)}}\left(\sum_{k=0}^{n} A^{(k)} \boldsymbol{d} \psi_{k}\right)^{2} .
\end{aligned}
$$

Here $c$ is a conventional constant. ${ }^{3}$ We can again introduce the frames of 1 -forms given by (2.5) completed with 1 -forms which we label by the index $\hat{0} \equiv 2 n+1$

$$
\boldsymbol{\epsilon}^{\hat{0}}=\sum_{k=0}^{n} A^{(k)} \boldsymbol{d} \psi_{k}, \quad \boldsymbol{e}^{\hat{0}}=\left[\frac{c}{A^{(n)}}\right]^{1 / 2} \boldsymbol{\epsilon}^{\hat{0}} .
$$

The dual frames then become

$$
\begin{array}{rlrl}
\boldsymbol{\epsilon}_{\hat{\mu}} & =\sum_{k=0}^{n} \frac{\left(-x_{\mu}^{2}\right)^{n-1-k}}{U_{\mu}} \boldsymbol{\partial}_{\psi_{k}}, & \boldsymbol{e}_{\hat{\mu}}=\left[\frac{U_{\mu}}{X_{\mu}}\right]^{1 / 2} \boldsymbol{\epsilon}_{\hat{\mu}} \\
\boldsymbol{\epsilon}_{\hat{0}}=\frac{1}{A^{(n)}} \boldsymbol{\partial}_{\psi_{n}}, & \boldsymbol{e}_{\hat{0}}=\left[\frac{A^{(n)}}{c}\right]^{1 / 2} \boldsymbol{\epsilon}_{\hat{0}} .
\end{array}
$$

The metric diagonalizes

$\boldsymbol{g}=\sum_{\mu=1}^{n}\left(\frac{U_{\mu}}{X_{\mu}} \boldsymbol{\epsilon}^{\mu} \boldsymbol{\epsilon}^{\mu}+\frac{X_{\mu}}{U_{\mu}} \boldsymbol{\epsilon}^{\hat{\mu}} \boldsymbol{\epsilon}^{\hat{\mu}}\right)+\frac{c}{A^{(n)}} \boldsymbol{\epsilon}^{\hat{0}} \boldsymbol{\epsilon}^{\hat{0}}=\sum_{a=1}^{D} \boldsymbol{e}^{a} \boldsymbol{e}^{a}$,

\footnotetext{
3 The constant $c$ could be eliminated by a appropriate rescaling of the coordinates and other parameters.
}

as well as the the Ricci tensor [7]

$$
\operatorname{Ric}=-\sum_{\mu=1}^{n} r_{\mu}\left(e^{\mu} e^{\mu}+e^{\hat{\mu}} e^{\hat{\mu}}\right)-r_{0} e^{\hat{0}} e^{\hat{0}}
$$

Here

$$
\begin{aligned}
r_{\mu} & =\frac{1}{2} \frac{\bar{X}_{\mu}^{\prime \prime}}{U_{\mu}}+\frac{1}{2 x_{\mu}} \frac{\bar{X}_{\mu}^{\prime}}{U_{\mu}}+\sum_{\substack{\nu=1 \\
\nu \neq \mu}}^{n} \frac{1}{U_{\nu}} \frac{x_{\nu} \bar{X}_{\nu}^{\prime}-x_{\mu} \bar{X}_{\mu}^{\prime}}{x_{\nu}^{2}-x_{\mu}^{2}} \\
& =\frac{\partial}{\partial x_{\mu}^{2}}\left[\sum_{\nu=1}^{n} \frac{x_{\nu} \bar{X}_{\nu}^{\prime}}{U_{\nu}}\right], \\
r_{0} & =\sum_{\nu=1}^{n} \frac{\bar{X}_{\nu}^{\prime}}{x_{\nu} U_{\nu}}
\end{aligned}
$$

and we used shifted metric functions

$$
\bar{X}_{\mu}=X_{\mu}+\frac{c}{x_{\mu}^{2}} .
$$

The scalar curvature becomes

$$
\mathcal{R}=-\sum_{\nu=1}^{n} \frac{\bar{X}_{\nu}^{\prime \prime}}{U_{\nu}}-2 \sum_{\nu=1}^{n} \frac{1}{x_{\nu}} \frac{\bar{X}_{\nu}^{\prime}}{U_{\nu}} .
$$

Finally, the vacuum Einstein equations require

$$
X_{\mu}=-\frac{c}{x_{\mu}^{2}}+b_{\mu}+\sum_{k=0}^{n-1} c_{k} x_{\mu}^{2 k},
$$

The spacetime has $n+1$ Killing vectors $\boldsymbol{\partial}_{\psi_{j}}$ and there exists the principal Killing-Yano tensor dual to the conformal Killing-Yano tensor which is given again by (2.11).

\section{Algebraically special test electromagnetic field}

As in the even dimensions we look for the electromagnetic field with the structure given by (2.12). On could ask if in the odd dimension couldn't be this ansatz extended by an additional term related to the 'unpaired' direction, i.e., by the term of the form $f_{0} \boldsymbol{d} x \wedge \boldsymbol{\epsilon}^{\hat{0}}$ with functions $f_{0}$ and $x$ independent of the coordinates $\psi_{j}$. However, we do not consider such terms since they lead to some unwanted consequences (e.g., they lead to a nondiagonal stress-energy tensor).

It follows from the first Maxwell equations that the vector potential can be written as

$$
\boldsymbol{A}=\frac{g_{0}}{A^{(n)}} \boldsymbol{\epsilon}^{\hat{0}}+\sum_{\mu=1}^{n} \frac{g_{\mu}}{U_{\mu}} \boldsymbol{\epsilon}^{\hat{\mu}},
$$

where $g_{\mu}$ is a function of $x_{\mu}$ only and $g_{0}$ is a constant. The components of the Maxwell tensor can be again generated by (2.16) from the auxiliary potential

$$
\phi=\frac{g_{0}}{A^{(n)}}+\sum_{\nu=1}^{n} \frac{g_{\nu}}{U_{\nu}}
$$


and in terms of $g$ 's they take the form

$$
f_{\mu}=\frac{g_{\mu}^{\prime}}{U_{\mu}}+2 x_{\mu} \sum_{\substack{\nu=1 \\ \nu \neq \mu}}^{n} \frac{1}{U_{\nu}} \frac{g_{\nu}-g_{\mu}}{x_{\nu}^{2}-x_{\mu}^{2}}-\frac{2 g_{0}}{x_{\mu} A^{(n)}} .
$$

The electromagnetic current becomes

$$
\boldsymbol{J}=-2 \sum_{\mu=1}^{n} \frac{\partial}{\partial x_{\mu}^{2}}\left[x_{\mu}^{2} \sum_{\nu=1}^{n} x_{\mu}^{-1} \phi_{\nu}\right] \boldsymbol{\epsilon}_{\hat{\mu}}+2 \sum_{\nu=1}^{n} \frac{1}{x_{\mu}} \phi_{, \nu} \boldsymbol{\epsilon}_{\hat{0}} .
$$

Solving the second Maxwell equation without sources (i.e., $\boldsymbol{J}=0$ ) we obtain

$$
g_{\mu}=e_{\mu}-\frac{g_{0}}{x_{\mu}^{2}}+\sum_{k=0}^{n-2} a_{k}\left(-x_{\mu}^{2}\right)^{n-1-k} .
$$

The last two terms are gauge trivial and the vector potential can thus be written as

$$
\boldsymbol{A}=\sum_{\mu=1}^{n} \frac{e_{\mu}}{U_{\mu}} \epsilon^{\hat{\mu}},
$$

and the auxiliary potential $\phi$ as

$$
\phi=\sum_{\nu=1}^{n} \frac{e_{\nu}}{U_{\nu}} .
$$

Finally, for the components of the Maxwell tensor we find

$$
f_{\mu}=2 x_{\mu} \sum_{\nu=1}^{n} \frac{1}{U_{\nu}} \frac{e_{\nu}-e_{\mu}}{x_{\nu}^{2}-x_{\mu}^{2}} .
$$

\section{The Chern-Simons term}

In an odd number of dimensions we can consider Chern-Simons modification of the electromagnetic field. Since the Chern-Simons term in the action does not refer to the metric, it does not change the Einstein equation and the stress-energy tensor of the electromagnetic field. However, it modifies the divergence $-\boldsymbol{\nabla} \cdot \boldsymbol{F}$ in the second Maxwell equation by adding a non-linear term $\boldsymbol{J}_{\mathrm{ChS}}$ proportional (with a constant coefficient) to the Hodge dual of the $n$-th wedge power of $\boldsymbol{F}$,

$$
\boldsymbol{J}_{\mathrm{ChS}} \propto *(\underbrace{\boldsymbol{F} \wedge \cdots \wedge \boldsymbol{F}}_{n \text { times }}) .
$$

Since the Levi-Civita tensor $\varepsilon$ used in the Hodge dual is given by the product $\boldsymbol{e}^{1} \wedge \cdots \wedge \boldsymbol{e}^{2 n+1}$ we find that the Chern-Simons term $\boldsymbol{J}_{\mathrm{ChS}}$ for the field (2.12) is aligned with the 'unpaired' direction

$$
\boldsymbol{J}_{\mathrm{ChS}} \propto\left[\prod_{\mu=1}^{n} f_{\mu}\right] \boldsymbol{e}_{\hat{0}} .
$$

We thus have to solve the Maxwell equation $\boldsymbol{J}=\boldsymbol{J}_{\mathrm{ChS}}$ with $\boldsymbol{J}$ given by (3.13). The condition that $\boldsymbol{J}$ vanishes in $\boldsymbol{e}_{\hat{\mu}}, \mu=1, \ldots, n$, directions is only slightly weaker than that we solved in the previous section. We find that the vector potential $\boldsymbol{A}$, the auxiliary potential $\phi$, and the components of the electromagnetic field are

$$
\begin{aligned}
\boldsymbol{A} & =\sum_{\mu=1}^{n} \frac{e_{\mu}+a \log x_{\mu}^{2}}{U_{\mu}} \boldsymbol{\epsilon}^{\hat{\mu}}, \quad \phi=\sum_{\nu=1}^{n} \frac{e_{\nu}+a \log x_{\mu}^{2}}{U_{\nu}} \\
f_{\mu} & =2 x_{\mu} \sum_{\nu=1}^{n} \frac{1}{U_{\nu}} \frac{e_{\nu}-e_{\mu}}{x_{\nu}^{2}-x_{\mu}^{2}} \\
& +2 a\left[\frac{1}{x_{\mu}}+x_{\mu} \sum_{\substack{\nu=1 \\
\nu \neq \mu}}^{n} \frac{1}{U_{\nu}} \frac{\log x_{\nu}^{2}-\log x_{\mu}^{2}}{x_{\nu}^{2}-x_{\mu}^{2}}\right]
\end{aligned}
$$

where $a$ is a constant parameter. Moreover, the source term $\boldsymbol{J}$ must be

$$
\boldsymbol{J}=\frac{4 a}{A^{(n)}} e^{\hat{0}} .
$$

Although we did not prove it rigorously it seems evident that this component cannot be equal to (3.19) with $f_{\mu}$ given by (3.20). We thus conclude that the ChernSimons modification of the algebraically special electromagnetic field is not possible.

\section{Einstein-Maxwell equations in odd $D$}

The stress-energy tensor for the field (2.12) in odd dimensions is also diagonal (with the additional component $8 \pi T_{\hat{0} \hat{0}}=-f^{2}$ ). The condition for the scalar curvature following from the Einstein equations is again given by (2.33), and by the same argument as in the even dimensions (now with help of (3.8) ) we conclude that the algebraically special electromagnetic field (2.12), (3.17) cannot couple to the metric given by (3.1).

\section{SUMMARY}

We have found explicitly the test electromagnetic field on the background of the high-dimensional rotating NUT charged black hole [6]. The field solves the Maxwell equations even in a broader class of spacetimes since the specific form of the metric functions $X_{\mu}$ is not needed. The common feature of these spacetimes is the presence of $\lceil D / 2\rceil$ Killing vectors and the existence of the principal Killing-Yano tensor [9]. The constructed electromagnetic field is adjusted to this structure - it shares the explicit symmetries and it has the same eigenspaces as the principal conformal Killing-Yano tensor.

The electromagnetic field depends on $n=\lfloor D / 2\rfloor$ constants $e_{\mu}$ related to the global electric and magnetic charges. It generalizes the field known on the background of the Plebański-Demiański spacetime in $D=4$ dimensions. In this case the metric functions can be modified 
in such a way that the field and the metric solve the full Einstein-Maxwell equations. Unfortunately, an analogous modification is not possible in a generic dimension.

Finally, we have shown that the Chern-Simons generalization in an odd dimmension is also not permitted for the electromagnetic field of this form.

\section{Acknowledgments}

This work was supported by the grant GAČR 202/06/0041 and by the Czech Ministry of Education under the project LC06014. The author would like to thank Don N. Page, Valeri P. Frolov, David Kubizňák and Jiří Podolský for numerous discussions, and Jiří Podolský and David Kubizñák for reading the manuscript.

\section{APPENDIX A}

The functions $A_{\mu}^{(k)}$ and $U_{\mu}$ defined in (2.2) satisfy the following useful identities

$$
\begin{gathered}
\sum_{\mu=1}^{n} A_{\mu}^{(i)} \frac{\left(-x_{\mu}^{2}\right)^{n-1-j}}{U_{\mu}}=\delta_{j}^{i}, \quad \sum_{j=0}^{n-1} A_{\mu}^{(j)} \frac{\left(-x_{\nu}^{2}\right)^{n-1-j}}{U_{\nu}}=\delta_{\mu}^{\nu} \\
\sum_{\mu=1}^{n} A_{\mu}^{(i)} \frac{\left(-x_{\mu}^{2}\right)^{n}}{U_{\mu}}=-A^{(j+1)}, \quad \sum_{\mu=1}^{n} \frac{A_{\mu}^{(j)}}{x_{\mu}^{2} U_{\mu}}=\frac{A^{(j)}}{A^{(n)}}, \\
\sum_{j=0}^{n}(n-j) A^{(j)} \frac{\left(-x_{\mu}^{2}\right)^{n-1-j}}{U_{\mu}}=1, \\
\sum_{j=0}^{n-1}(n-1-j) A_{\mu}^{(j)} \frac{\left(-x_{\mu}^{2}\right)^{n-1-j}}{U_{\mu}}=\sum_{\substack{\nu=1 \\
\nu \neq \mu}}^{n} \frac{x_{\mu}^{2}}{x_{\mu}^{2}-x_{\nu}^{2}}, \quad(\mathrm{~A} 2) \\
\sum_{j=0}^{n-1}(n-1-j) A_{\nu}^{(j)} \frac{\left(-x_{\mu}^{2}\right)^{n-1-j}}{U_{\mu}}=\frac{x_{\mu}^{2}}{x_{\mu}^{2}-x_{\nu}^{2}} \quad \text { for } \mu \neq \nu
\end{gathered}
$$

and

$$
\frac{1}{U_{\mu}} \sum_{\substack{\nu=1 \\ \nu \neq \mu}}^{n} \frac{1}{x_{\nu}^{2}-x_{\mu}^{2}}=-\sum_{\substack{\nu=1 \\ \nu \neq \mu}}^{n} \frac{1}{U_{\nu}} \frac{1}{x_{\nu}^{2}-x_{\mu}^{2}}
$$

We list also external derivatives of the function $1 / U_{\mu}$ and of the 1-form $\epsilon^{\hat{\mu}}$ which have been used repeatedly in the computations:

$$
\begin{gathered}
\boldsymbol{d} \frac{1}{U_{\mu}}=-\frac{1}{U_{\mu}} \sum_{\substack{\nu=1 \\
\nu \neq \mu}}^{n} \frac{2}{x_{\nu}^{2}-x_{\mu}^{2}}\left(x_{\nu} \boldsymbol{\epsilon}^{\nu}-x_{\mu} \boldsymbol{\epsilon}^{\mu}\right), \\
\boldsymbol{d} \boldsymbol{\epsilon}^{\hat{\mu}}=\sum_{\substack{\nu=1 \\
\nu \neq \mu}}^{n} \frac{2}{x_{\nu}^{2}-x_{\mu}^{2}}\left(x_{\nu} \boldsymbol{\epsilon}^{\nu} \wedge \boldsymbol{\epsilon}^{\hat{\mu}}-x_{\nu} \boldsymbol{\epsilon}^{\nu} \wedge \boldsymbol{\epsilon}^{\hat{\nu}}\right) .
\end{gathered}
$$

Finally, let us formulate explicitly an important lemma (which has been already used implicitly in [7]) concerning properties of the sums $\sum_{\nu} h_{\nu} / U_{\nu}$. Let's consider the equation

$$
\sum_{\mu=1}^{n} \frac{h_{\mu}}{U_{\mu}}=0
$$

where $h_{\mu}$ are functions of a single variable $x_{\mu}$ only. Then these functions are given by a single polynomial

$$
h_{\mu}=\sum_{k=0}^{n-2} c_{k} x_{\mu}^{2 k}
$$

with arbitrary coefficients $c_{k}, k=0, \ldots, n-2$.

The fact that the polynomial functions $\mathrm{A} 6 \mathrm{~b}$ solve equation (A6a) follows from (A1). The opposite implication is less trivial - first one has to show that $h_{\mu}$ must be polynomials of the order $n-2$ (by differentiating repeatedly $\left.U_{\mu} \sum_{\nu} h_{\nu} / U_{\nu}\right)$. Next, a more intricate task is to prove that the polynomials for different $\mu$ are the same. It can be achieved by an induction in $n$.

The solution of the functional equation A6a with a non-trivial right-hand-side is then given by the sum of a particular solution with the homogeneous solution (A6b). We mention three important particular solutions for simple right-hand-side terms:

$$
\begin{gathered}
\sum_{\mu=1}^{n} \frac{\left(-x_{\mu}^{2}\right)^{n-1}}{U_{\mu}}=1, \\
\sum_{\mu=1}^{n} \frac{\left(-x_{\mu}^{2}\right)^{n}}{U_{\mu}}=-A^{(1)}, \\
\sum_{\mu=1}^{n} \frac{1}{x_{\mu}^{2} U_{\mu}}=\frac{1}{A^{(n)}},
\end{gathered}
$$

all following from the relations (A1).

The property (A6) has been used, for example, to derive the specific forms (2.10) and (3.9) of the metric functions $X_{\mu}$ starting from the condition $\mathcal{R}=$ constant with the scalar curvature given by (2.9) or (3.8). It has been used also to find the components (2.22) and (3.14) of the vector potential. 
[1] F. R. Tangherlini, Schwartzschild Field in $N$ Dimensions and the Dimensionality of Space Problem, Nuovo Cimento 27, 3 (1963).

[2] R. C. Myers and M. J. Perry, Black holes in higher dimensional space-times, Ann. Phys. (N.Y.) 172, 304 (1986).

[3] S. W. Hawking, C. J. Hunter, and M. M. TaylorRobinson, Rotation and the AdS/CFT correspondence, Phys. Rev. D 59, 064005 (1999), arXiv:hep-th/9811056

[4] G. W. Gibbons, H. Lü, D. N. Page, and C. N. Pope, Rotating Black Holes in Higher Dimensions with a Cosmological Constant, Phys. Rev. Lett. 93, 171102 (2004), arXiv:hep-th/0409155.

[5] G. W. Gibbons, H. Lü, D. N. Page, and C. N. Pope, The General Kerr-de Sitter Metrics in All Dimensions, J. Geom. Phys. 53, 49 (2005), arXiv:hep-th/0404008

[6] W. Chen, H. Lü, and C. N. Pope, General Kerr-NUTAdS metrics in all dimensions, Class. Quantum Grav. 23, 5323 (2006), arXiv:hep-th/0604125.

[7] N. Hamamoto, T. Houri, T. Oota, and Y. Yasui, KerrNUT-de Sitter curvature in all dimensions, J. Phys. A40, F177 (2007), arXiv:hep-th/0611285.

[8] D. Kubizńák and V. P. Frolov, Hidden symmetry of higher dimensional Kerr-NUT-AdS spacetimes, Class. Quantum Grav. 24, F1 (2007), arXiv:gr-qc/0610144.

[9] P. Krtouš, D. Kubizňák, D. N. Page, and V. P. Frolov, Killing-Yano tensors, rank-2 Killing tensors, and conserved quantities in higher dimensions, J. High Energy Phys. JHEP02(2007)004, arXiv:hep-th/0612029.

[10] D. N. Page, D. Kubizňák, M. Vasudevan, and P. Krtous, Integrability of geodesic motion in general Kerr-NUTAdS spacetimes, Phys. Rev. Lett. 98, 061102 (2007), arXiv:hep-th/0611083.

[11] P. Krtouš, D. Kubizňák, D. N. Page, and M. Vasudevan, Constants of Geodesic Motion in Higher-Dimensional Black-Hole Spacetimes, arXiv:0707.0001 [hep-th].

[12] V. P. Frolov, P. Krtouš, and D. Kubizńák, Separability of Hamilton-Jacobi and Klein-Gordon equations in general Kerr-NUT-AdS spacetimes, J. High Energy Phys. JHEP02(2007)005, arXiv:hep-th/0611245.

[13] A. N. Aliev and V. P. Frolov, Five dimensional rotating black hole in a uniform magnetic field: The gyromagnetic ratio, Phys. Rev. D 69, 084022 (2004), arXiv:hep-th/0401095.
[14] A. N. Aliev, Charged Slowly Rotating Black Holes in Five Dimensions, Mod. Phys. Lett. A21, 751 (2006), arXiv:gr-qc/0505003

[15] A. N. Aliev, Rotating black holes in higher dimensional Einstein-Maxwell gravity, Phys. Rev. D 74, 024011 (2006), arXiv:hep-th/0604207.

[16] A. N. Aliev, Electromagnetic Properties of Kerr-Antide Sitter Black Holes, Phys. Rev. D 75, 084041 (2007), arXiv:hep-th/0702129.

[17] Y. Brihaye and T. Delsate, Charged-rotating black holes and black strings in higher dimensional Einstein-Maxwell theory with a positive cosmological constant, 2007, arXiv:gr-qc/0703146

[18] J. Kunz, F. Navarro-Lerida, and E. Radu, Higher dimensional rotating black holes in Einstein-Maxwell theory with negative cosmological constant, Phys. Lett. B649, 463 (2007), arXiv:gr-qc/0702086.

[19] W. Chen and H. Lü, Kerr-Schild Structure and Harmonic 2-forms on (A)dS-Kerr-NUT Metrics, 2007, arXiv:0705.4471 [hep-th].

[20] B. Carter, Hamilton-Jacobi and Schrodinger Separable Solutions of Einstein's Equations, Commun. Math. Phys. 10, 280 (1968).

[21] J. Plebański and M. Demiański, Rotating charged and uniformly accelerated mass in general relativity, Ann. Phys. (N.Y.) 98, 98 (1976).

[22] J. B. Griffiths and J. Podolský, Accelerating and rotating black holes, Class. Quantum Grav. 22, 3467 (2005), arXiv:gr-qc/0507021.

[23] J. B. Griffiths and J. Podolský, Global aspects of accelerating and rotating black hole space-times, Class. Quantum Grav. 23, 555 (2006), arXiv:gr-qc/0511122.

[24] J. B. Griffiths and J. Podolský, A new look at the Plebanski-Demianski family of solutions, Int. J. Mod. Phys. D15, 335 (2006), arXiv:gr-qc/0511091.

[25] J. Podolský and J. B. Griffiths, Accelerating KerrNewman black holes in (anti-)de Sitter space-time, Phys. Rev. D 73, 044018 (2006), arXiv:gr-qc/0601130.

[26] J. B. Griffiths and J. Podolský, On the parameters of the Kerr-NUT-(anti-)de Sitter space-time, Class. Quantum Grav. 24, 1687 (2007), arXiv:gr-qc/0702042 